\title{
Trivia quiz
}

Matthew Jelley and John Owen

\section{Mental Health Act Addendum}

Section 12 Approval (Supplementary Test)

All doctors applying for approval under Section 12 MHA (1983) must now display their knowledge by answering the following questions.

1 What have the following in common: "Asylum", "Going Under", and "Egg Dancing"?

2 Who played a theatre in Leicester Square in 1897 and subsequently made an impression on psychiatry?

3 What was the A.M.O.A.H.I.?

4 Who was The President's Analyst in 1967?
5 Who stated that they were "not so manic now" last year?

6 In The Final Analysis my patient, Mr Jones, became a therapist. Who am I?

7 Who stated "If you talk to God, you are praying; if God talks to you, you have schizophrenia"?

8 What was Edward Drummond's job in 1843?

9 Which psychiatrist has a Green River Rising and some Blood-Stained Kings?

10 Who played Don Juan de Marcos' therapist?

11 What does A Person Meditating on Madness have in his right hand?

12 What are the merergasias? 